\title{
On the properties of the transition matrix in bouncing cosmologies
}

\author{
Jérôme Martin* and Patrick Peten ${ }^{\dagger}$ \\ Institut d'Astrophysique de Paris, $\mathcal{G} \mathbb{R} \mathbb{C O}$, FRE 2435-CNRS, 98bis boulevard Arago, 75014 Paris, France
}

(Dated: January $27^{\text {th }}, 2004$ )

\begin{abstract}
We elaborate further on the evolution properties of cosmological fluctuations through a bounce. We show this evolution to be describable either by "transmission" and "reflection" coefficients or by an effective unitary $S$-matrix. We also show that they behave in a time reversal invariant way. Therefore, earlier results are now interpreted in a different perspective and put on a firmer basis.
\end{abstract}

PACS numbers: $98.80 . \mathrm{Cq}, 98.70 . \mathrm{Vc}$

\section{INTRODUCTION}

A (Jordan or Einstein frame-) bounce [1] is predicted to have taken place in some string - or quantum gravity - motivated early universe models, as e.g. the pre big bang 2] and ekpyrotic cases 3, 4]. Those models are often presented as plausible challengers to the otherwise well-confirmed inflation paradigm, and must therefore be able to provide an almost scale-invariant perturbation spectrum.

The difficulty resides in the propagation of the spectrum, usually produced in the contracting phase, through the bounce, as the latter often involves high curvatures and thus knowledge of at least some non-linear terms in the underlying theory. Even assuming that the perturbations can in fact somehow propagate through, i.e. supposing the first order to remain meaningful all along, a point quite debatable in itself (see e.g. Lyth in Refs. 4]), it is necessary to impose a specific model to examine the validity of the usual assumptions that are made, either explicitly or implicitly, in the abovementionned frameworks.

This has been done in Refs. [5, 6] in which we used pure general relativity, a Friedmann-Robertson-Walker metric with positively curved spatial sections and a scalar field $\varphi$ to provide the matter content and lead the dynamics of the scale factor $a(\eta)$ as a function of conformal time $\eta$. We have since received inquiries about some seemingly unexpected features of the results, such as an apparently vanishing-determinant transition matrix, and we were questioned about the unitarity of the evolution.

In this brief report we clarify these issues by showing that, since the evolution equation can be understood as a time-independent Schrödinger equation, its solutions can be interpreted as transmission and reflection coefficients, which ensures the conservation of probability, or, stated differentely, the unitarity of the $S$-matrix that can be built out of the transition. Note however that these results stem from the mathematical properties of the Schrödinger equation, although the actual interpretation of the underlying classical perturbed Einstein equa-

\footnotetext{
*Electronic address: jmartin@iap.fr
}

$\dagger$ Electronic address: peter@iap.fr tions does involve neither any physical wavefunction nor any probabilistic meaning. In particular, this should not be confused with the effective quantization of the physical modes, i.e. the second quantization of the semi-classical effective field theory which underpins inflationary structure formation scenarios.

In addition, we show that the transition matrix reflects the underlying invariance under time reversal. Finally, we also demonstrate that the vanishing of the determinant signaled before was to be expected and turns out in fact to provide a necessary consistency check of the calculations performed in Ref. [5].

\section{THE BOUNCE SEEN AS A POTENTIAL OF A SCHRÖDINGER EQUATION}

We want to describe the evolution of cosmological perturbations in a universe with positively curved spatial sections through a bounce described by general relativity and a scalar field $\phi$. The scale factor $a$ depends on the conformal time $\eta$. One can construct a perturbation function $u \propto \Phi$, proportional to the Bardeen potential $\Phi$ through a function of the background 5] which we do not need to specify here, and satisfying [5, 6 ]

$$
u^{\prime \prime}+\left[k^{2}-V_{u}(\eta)\right] u=0,
$$

for a wavenumber $k=\sqrt{n(n+2)}$ on the 3-sphere. By setting $k^{2} \rightarrow E, u \rightarrow \psi$ and $\eta \rightarrow \sqrt{2 m} x / \hbar$, Eq. (1) is of the form of a time-independent Schrödinger equation $-\left(\hbar^{2} / 2 m\right) \mathrm{d}^{2} \psi / \mathrm{d} x^{2}+[V(x)-E] \psi=0$ in the variable $\eta$ for the "wavefunction" $u$ in the potential $V_{u}(x)$. Given this form, the current $\mathcal{J}=$ $-(i \hbar / 2 m)\left(\psi^{\star} \nabla \psi-\psi \nabla \psi^{\star}\right) \rightarrow-i\left(u^{\star} u^{\prime}-u u^{\star \prime}\right)$ is conserved $\nabla \cdot \mathcal{J}=0 \rightarrow \mathcal{J}^{\prime}=0$.

In the case of a symmetric bounce, the potential $V_{u}(\eta)$ of Eq. (1) is nonvanishing in a finite and symmetric region around $\eta=0$, i.e. for $-\eta_{\mathrm{z}} \leq \eta \leq x_{\mathrm{z}}$, which we shall call region II, surrounded by regions I (for $\eta$ before the bounce) and III (for $\eta$ after the bounce). In both regions I and III, the potential being negligible, we have

$$
u_{\mathrm{I}, \mathrm{III}}=\frac{1}{\sqrt{2 k}}\left(A_{\mathrm{I}, \mathrm{III}} \mathrm{e}^{-i k \eta}+B_{\mathrm{I}, \mathrm{III}} \mathrm{e}^{i k \eta}\right),
$$



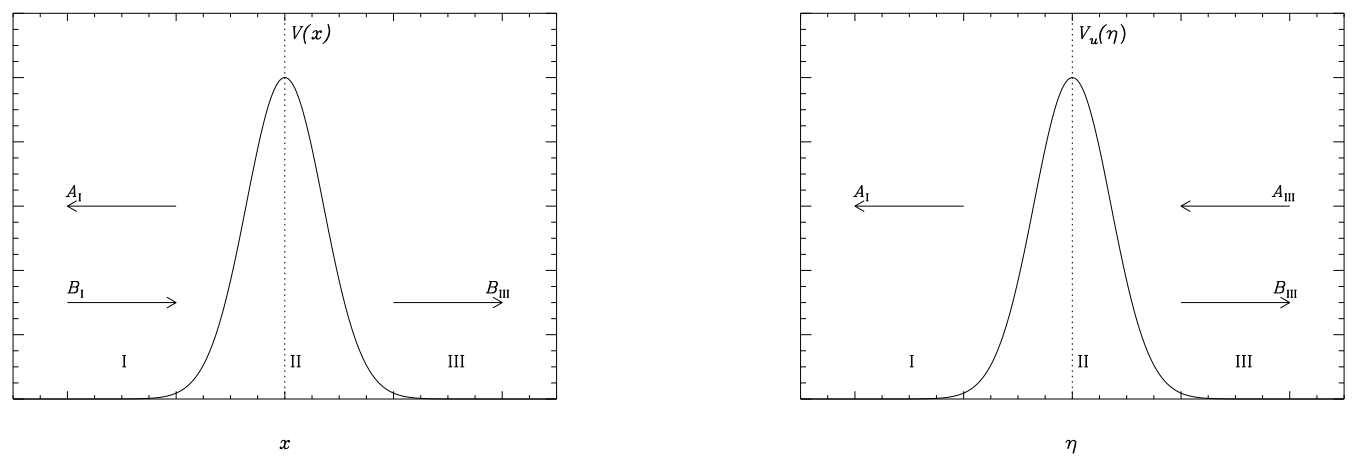

FIG. 1: The analogy and differences between the wavefunction across a potential in ordinary quantum mechanics and primordial perturbations through a bounce. In both cases, the total current is conserved.

leading to the currents

$$
J_{\mathrm{I}, \mathrm{III}}=\left|B_{\mathrm{I}, \mathrm{III}}\right|^{2}-\left|A_{\mathrm{I}, \mathrm{III}}\right|^{2}
$$

on both sides of the bounce. Apart from the overall irrelevant normalization, this is understood, in ordinary quantum mechanics, as the difference between current probabilities of wavefunctions traveling respectively in the $-\eta$ (coefficient $A$ ) and $+\eta$ (coefficient $B$ ) directions. The transition matrix relating $\left(A_{\mathrm{III}}, B_{\mathrm{III}}\right)$ to $\left(A_{\mathrm{I}}, B_{\mathrm{I}}\right)$ was obtained in Refs. [5, 6] by expanding the solution in the region II into an even $f_{\mathrm{II}}(-\eta)=f_{\mathrm{II}}(\eta)$ and an odd $\varnothing(-\eta)=-\varnothing(-\eta)$ functions, which can always be done in the case of a symmetric potential.

Setting $f_{\mathrm{II}} \equiv f_{\mathrm{II}}\left(\eta_{\mathrm{z}}\right), f_{\mathrm{II}}^{\prime} \equiv \mathrm{d} f_{\mathrm{II}}\left(\eta_{\mathrm{z}}\right) / \mathrm{d} \eta, \varnothing \equiv \varnothing\left(\eta_{\mathrm{z}}\right)$ and $\varnothing^{\prime} \equiv \mathrm{d} \varnothing\left(\eta_{\mathrm{z}}\right) / \mathrm{d} \eta$, we found

$$
\left(\begin{array}{l}
A_{\mathrm{III}} \\
B_{\mathrm{III}}
\end{array}\right)=\left(\begin{array}{cc}
X & Y \\
Y^{\star} & X^{\star}
\end{array}\right)\left(\begin{array}{l}
A_{\mathrm{I}} \\
B_{\mathrm{I}}
\end{array}\right) \equiv T_{u}\left(\begin{array}{l}
A_{\mathrm{I}} \\
B_{\mathrm{I}}
\end{array}\right)
$$

thereby defining the transition matrix $T_{u}$, where

$$
\begin{aligned}
X & =\frac{i \mathrm{e}^{2 i k x_{\mathrm{z}}}}{k} \frac{\left(f_{\mathrm{II}}^{\prime}-i k f_{\mathrm{II}}\right)\left(\emptyset^{\prime}-i k \emptyset\right)}{\left(f_{\mathrm{II}} \emptyset^{\prime}-\emptyset f_{\mathrm{II}}^{\prime}\right)}, \\
Y & =\frac{i}{k} \frac{\left(f_{\mathrm{II}}^{\prime} \varnothing^{\prime}+k^{2} \varnothing f_{\mathrm{II}}\right)}{\left(f_{\mathrm{II}} \varnothing^{\prime}-\varnothing f_{\mathrm{II}}^{\prime}\right)},
\end{aligned}
$$

both terms being calculated at the points of matching.

From Eq. (4), one sees that the determinant of $T_{u}$ is unity, namely $|X|^{2}-|Y|^{2}=1$, as may be checked by direct calculation. This property is related to the fact that the propagation of perturbations through a bounce is similar to that of a wavefunction $\psi(x)$ through a potential $V(x)$, as illustrated on the right of Fig. 1 If the incoming wavefunction has amplitude $B_{\mathrm{I}}$, it is reflected with amplitude $A_{\mathrm{I}}$, leading to a reflection coefficient $R=\left|A_{\mathrm{I}} / B_{\mathrm{I}}\right|^{2}$, and the amount $B_{\mathrm{III}}$ goes through, so that the transmission coefficient is $T=\left|B_{\mathrm{III}} / B_{\mathrm{I}}\right|^{2}$ (assuming the same wavenumber on both sides). For the bounce case shown on the left, the analogy makes sense provided one reads the figure backwards in time. Then, the "transmitted" wave, with amplitude $A_{\mathrm{I}}$, in fact the original perturbation, travels again in the same direction as the wave hitting the potential with amplitude $B_{\mathrm{III}}$, leading to a transmission coefficient $T_{\mathrm{B}} \equiv\left|A_{\mathrm{I}} / A_{\mathrm{III}}\right|^{2}$, while the reflection coefficient reads $R_{\mathrm{B}} \equiv\left|B_{\mathrm{III}} / A_{\mathrm{III}}\right|^{2}$. Then, the current conservation, $\mathcal{J}^{\prime}=0$, implies that

$$
R_{\mathrm{B}}+T_{\mathrm{B}}=1,
$$

as expected. Notice that this is just a rewriting of the matching condition $\left|B_{\mathrm{III}}\right|^{2}-\left|A_{\mathrm{III}}\right|^{2}=\left|B_{\mathrm{II}}\right|^{2}-\left|A_{\mathrm{I}}\right|^{2}$, itself a consequence of the fact that the transition matrix is of unit determinant: this merely expresses the conservation of probability in ordinary quantum mechanics. The result obtained in Ref. [5] is therefore in perfect agreement with the conservation of the effective probability current associated with the otherwise classical Einstein equations.

\section{BOUNCE $S$-MATRIX AND UNITARITY}

Another way to express conservation of probability is through unitarity. Therefore, one must consider a different point of view, namely that of the $S$-matrix, to build the unitary transformation stemming from $T_{u}$. This is what we do in this section, by means of a comparison with standard collision processes in ordinary quantum mechanics. Obviously, there is no actual collision involved, and the fact that one can construct such a unitarity $S$-matrix merely reflects the mathematical consistency of Refs. [5, 6].

In three dimensional quantum mechanics, a plane wave propagating in an incident direction $\mathbf{n}=\mathbf{k} /|\mathbf{k}|$ and diffusing in a potential located at $O$ is observed at the point $M$ such that $O \vec{M} \equiv r \mathbf{n}^{\prime}$ leads to the collisional wave function $\Psi_{\mathbf{n}, \mathbf{n}^{\prime}}$ given by []

$$
\Psi_{\mathbf{n}, \mathbf{n}^{\prime}}=\mathrm{e}^{i k r \mathbf{n} \cdot \mathbf{n}^{\prime}}+\frac{1}{r} f_{\mathbf{n}, \mathbf{n}^{\prime}} \mathrm{e}^{i k r},
$$

thus defining the coefficients $f_{\mathbf{n}, \mathbf{n}^{\prime}}$ characterizing the diffusion and providing the $S$-matrix (see below), the fac- 
tor $1 / r$ in the second term stemming from the expression of a spherical wave in 3D. In a similar way, one can interpret the transformation Eq. (4) in terms of such a $S$-matrix, and hence to check unitarity.

In the cosmological one dimensional case, the $1 / r$ factor of Eq. (8) is absent, the role of the radial variable $r$ is played by the absolute value $|\eta|$ of the conformal time, and the unit vectors $\mathbf{n}$ and $\mathbf{n}^{\prime}$ can point towards only two directions, namely $+\hat{\eta}$ and $-\hat{\eta}$, so that $\mathbf{n} \cdot \mathbf{n}^{\prime}= \pm 1$. In what follows, we shall denote the time directions $\pm \hat{\eta}$ simply by the symbols + or - .

Let us consider the case of the figure on the right, i.e. the cosmological case with the potential $V_{u}(\eta)$. In this case, the analogous form of $\Psi$ for $u$ in Eq. (8) is $u_{\mathbf{n}, \mathbf{n}^{\prime}}$. After the bounce, the solution for $u$ is

$$
u_{\text {after }}=\frac{1}{\sqrt{2 k}}\left(A_{\mathrm{III}} \mathrm{e}^{-i k|\eta|}+B_{\mathrm{III}} \mathrm{e}^{i k|\eta|}\right),
$$

from which, in analogy with Eq. (8), one gets $\mathbf{n}=-\hat{\eta}$, $\mathbf{n}^{\prime}=+\hat{\eta}$ and therefore $f_{-+}$is simply the factor $B_{\mathrm{III}} / \sqrt{2 k}$ evaluated for $A_{\mathrm{III}}=\sqrt{2 k}$ and $B_{\mathrm{I}}=0$, i.e. $f_{-+}=Y^{\star} / X$, see Eq. (4). Before the bounce, $u$ is given by

$$
u_{\text {before }}=\frac{A_{\text {I }}}{\sqrt{2 k}} \mathrm{e}^{i k|\eta|}=\frac{1}{\sqrt{2 k}}\left[A_{\text {III }} \mathrm{e}^{i k|\eta|}+\left(A_{\mathrm{I}}-A_{\text {III }}\right) \mathrm{e}^{i k|\eta|}\right],
$$

from which, since one still has $\mathbf{n}=-\hat{\eta}$ but $\mathbf{n}^{\prime}=-\hat{\eta}$, the comparison with Eq. (8) provides the coefficient $f_{--}$, namely the value of $\left(A_{\mathrm{I}}-A_{\mathrm{III}}\right) / \sqrt{2 k}$ evaluated for $A_{\mathrm{III}}=$ $\sqrt{2 k}$, i.e. $f_{--}=1 / X-1$. Reasonning along the same line but in the reverse direction for $\mathbf{n}$ gives the coefficients $f_{++}=1 / X-1$ and $f_{+-}=-Y / X$.

The total wavefunction $\Psi(M)$ is the superposition of all the solutions for all incident directions weighted by arbitrary coefficients $F_{\mathbf{n}}$, i.e.

$$
\begin{aligned}
\Psi_{\mathbf{n}^{\prime}} & =\int F_{\mathbf{n}} \Psi_{\mathbf{n}, \mathbf{n}^{\prime}} \mathrm{d}^{3} \mathbf{n} \\
& \propto \mathrm{e}^{-i k r} F_{-\mathbf{n}^{\prime}}-\mathrm{e}^{i k r} S_{\mathbf{n}, \mathbf{n}^{\prime}} F_{\mathbf{n}^{\prime}},
\end{aligned}
$$

with which one defines the $S$-matrix as the coefficients in front of the outgoing modes. Similarly, in the bouncing case, one has the discrete sum

$$
u_{\mathbf{n}^{\prime}}=\sum_{\mathbf{n}= \pm \hat{\eta}} F_{\mathbf{n}} u_{\mathbf{n}, \mathbf{n}^{\prime}}
$$

leading to

$$
u_{+}=F_{-} \mathrm{e}^{-i k|\eta|}+\left[f_{-+} F_{-}+\left(1+f_{++}\right) F_{+}\right] \mathrm{e}^{i k|\eta|},
$$

and a similar formula by changing $\hat{\eta} \rightarrow-\hat{\eta}$. Gathering all the above results, this leads a two-by-two $S$-matrix that reads

$$
S=1+f=\frac{1}{X}\left(\begin{array}{cc}
1 & -Y \\
Y^{\star} & 1
\end{array}\right)
$$

which, because $|X|^{2}-|Y|^{2}=1$ as discussed in the previous sections, is unitary $\left(S S^{\dagger}=1\right)$. We have therefore built, from the non-unitary transformation $T_{u}$, the relevant unitary $S$-matrix, emphasizing once more the probability conservation.

\section{TIME INVERSION}

In Refs. [5, 6], we expanded the matrix $T_{u}$ of Eq. (4) in the parameter $\Upsilon$, which gives a measure of the deviation to the null energy condition $\rho+p \geq 0$ at the bounce. For $\Upsilon \ll 1$, the potential $V_{u}(\eta)$ goes to a function akin to a Dirac $\delta$ distribution, namely $V_{u}(\eta) \rightarrow-C_{\Upsilon} \delta(\eta)$, with $C_{\Upsilon} \propto \Upsilon^{-1 / 2} \gg 1$. With such a potential, the perturbation $u$ crosses the bounce unaltered, i.e. $[u]=0$, while its time derivative jumps by the amount $\left[u^{\prime}\right]=-C_{\Upsilon} u(0)$. These relations allow to obtain the matrix exactly, and we found

$$
T_{u}=\left(\begin{array}{cc}
1-i \frac{C_{\Upsilon}}{2 k} & -i \frac{C_{\Upsilon}}{2 k} \\
i \frac{C_{\Upsilon}}{2 k} & 1+i \frac{C_{\Upsilon}}{2 k}
\end{array}\right)=1-i \frac{C_{\Upsilon}}{2 k}\left(\begin{array}{cc}
1 & 1 \\
-1 & -1
\end{array}\right),
$$

which is indeed of the general form obtained in Eq. (44). We use this opportunity to correct a misprint in Eq. (77) of Ref. [5] in which the factor $i$ appears in the last term in the denominator whereas it should be at the numerator.

In the presentation made in Ref. [5], we explicitly assumed the limit $n(n+2) \Upsilon \ll 1$ and thus neglected the unit matrix part of $T_{u}$. Using Eq. (15), this gives

$$
T_{u}^{(\text {approx })}=-i \frac{C_{\Upsilon}}{2 k}\left(\begin{array}{cc}
1 & 1 \\
-1 & -1
\end{array}\right),
$$

in perfect agreement with Eq. (70) of Ref. [5]. As a consequence, the determinant of the above matrix vanishes

$$
\operatorname{det}\left[T_{u}^{(\text {approx })}\right]=0,
$$

whereas, in fact, the determinant of the exact matrix is unity, $\operatorname{det}\left(T_{u}\right)=1$.

With a time-symmetric potential $V_{u}(-\eta)=V(\eta)$ and only a second order time derivative term, one expects $T_{u}$ to be a time reversal invariant transformation. In other words, if the initial conditions are no longer fixed in the region I but in the region III, the final result, now expressed in region III and no longer in region I, should obviously be the same, in particular should still be proportional to $\Upsilon^{-1 / 2}$. If one wishes to check this property using $T_{u}^{\text {(approx) }}$, one faces the problem that it is not invertible. Hence, neglecting the term 1 in Eq. (15) has led to an apparent non symmetrical result while the problem clearly possesses this symmetry. The resolution of this paradox is obvious: the symmetry has been lost when 
the approximation has been performed and $T_{u}$, which is invertible since $\operatorname{det}\left(T_{u}\right) \neq 0$, should be used in this case. We are only authorized to neglect the term 1 in Eq. (15) if we want to obtain a close form of the transition matrix, as done in Ref. [5], but not if we want to check the time reversal invariance of the matrix. The fact that $\operatorname{det}\left[T_{u}^{(\text {approx })}\right]=0$ is actually nothing but a consistency check that the calculation performed in Ref. [5] is correct. Indeed, without this property, the inversion of $T_{u}^{\text {(approx) }}$ would have been possible, leading to an effect $\propto \Upsilon^{1 / 2}$ whereas the time reversal invariance implies that it should scale as $\Upsilon^{-1 / 2}$ in both time directions. The fact that $\operatorname{det}\left[T_{u}^{\text {(approx) }}\right]=0$ can therefore be interpreted as the "signal sent by the theory" that we are not allowed to use $T_{u}^{\text {(approx) }}$ to check the symmetry of the system.

It remains to be checked explicitly that the matrix $T_{u}$ has the correct time reversal symmetry. For this purpose, it is sufficient to calculate the inverse of $T_{u}$ and one obtains

$$
T_{u}^{-1}\left(C_{\Upsilon}\right)=T_{u}\left(-C_{\Upsilon}\right)
$$

Therefore, the amplitude of the transition matrix remains proportional to $\Upsilon^{-1 / 2}$ as required. The only difference is that the matrix of the inverse process should now be calculated with the parameter $-C_{\Upsilon}$ rather than $+C_{\Upsilon}$. The origin of this minus sign can easily be understood. Indeed, given the perturbation equation (1), time inversion( in the case at hand, given the analogy with the timeindependent Schrödinger equation, this operator is similar to parity) $\mathcal{T}$ manifests itself only in the matching condition on the first time derivative $u^{\prime}$ (which is not invariant) adding an extra minus sign, namely $\mathcal{T}\left(\left[u^{\prime}\right]\right)=-[u]$. This is exactly equivalent to changing the sign of $C_{\Upsilon}$. Comparing with Eq. (15), we see that

$$
\mathcal{T}\left[T_{u}\left(C_{\Upsilon}\right)\right]=T_{u}\left(-C_{\Upsilon}\right)=T_{u}^{-1}\left(C_{\Upsilon}\right),
$$

which merely expresses the familiar result that time inversion must satisfy $\mathcal{T}^{2}=\mathcal{T}$. Note that for an operator to be an arbitrary function of some parameter such as $C_{\Upsilon}$, the only way to ensure that $\mathcal{T}^{2}=\mathcal{T}$ is that $\mathcal{T}\left(C_{\Upsilon}\right)=-C_{\Upsilon}$ as indeed is the case here.

Finally, the explicit form of the $S$-matrix in the limit of Refs. [5, 6] is

$$
S=\frac{1}{1-\frac{i C_{\Upsilon}}{2 k}}\left(\begin{array}{cc}
1 & \frac{i C_{\Upsilon}}{2 k} \\
-\frac{i C_{\Upsilon}}{2 k} & 1
\end{array}\right) \simeq\left(\begin{array}{cc}
0 & -1 \\
1 & 0
\end{array}\right),
$$

where in the last approximation we have taken advantage of the condition $n(n+2) \Upsilon \ll 1$ as before.

\section{CONCLUSIONS}

We have presented a brief note on the propagation of primordial perturbations through a bouncing phase in the early universe to interpret this evolution in terms of either transmission and reflexion coefficients across the potential of a non-relativistic Schrödinger-like equation or in terms of the corresponding $S$-matrix that can be constructed out of the model. We have demonstrated that the results obtained in Refs. [5, 6] imply that $R_{\mathrm{B}}+T_{\mathrm{B}}=1$ and that the $S$-matrix is unitary, thereby checking the mathematical consistency of those results. Finally, we have also emphasized that the structure of the evolution equation is invariant under time reversal.

\section{Acknowledgments}

We are grateful to R. Brandenberger and B. Carter for many discussions that led to the above clarifications.
[1] R. Tolman, Phys. Rev. D 30, 1758 (1931); G. Lemaître, Ann. Soc. Sci. Bruxelles A 53, 5 (1933); A. Einstein, Sitzungsber., 235 (1931); P. Peter and N. PintoNeto,Phys. Rev. D 66, 063509 (2002); J. C. Fabris, R. G. Furtado, P. Peter, and N. Pinto-Neto, Phys. Rev. D 67, 124003 (2003); C. Cartier, R. Durrer, and E. J. Copeland, Phys. Rev. D 67, 103517 (2003); P. Peter and N. Pinto-Neto, JCAP 0312003 (2003); C. Gordon and N. Turok, Phys. Rev. D 67, 123508 (2003); M. Gasperini, M. Giovannini, and G. Veneziano, Phys. Lett. B 569, 113 (2003); hep-th/0401112

[2] See M. Gasperini and G. Veneziano, Phys. Rep. 373, 1 (2003) and references therein.

[3] J. Khoury, B. A. Ovrut, P. J. Steinhardt, and N. Turok, Phys. Rev. D 66, 046005 (2002) and references therein.
[4] R. Kallosh, L. Kofman, and A. Linde, Phys. Rev. D 64, 123523 (2001); D. H. Lyth, Phys. Lett. B 524, 1 (2002); R. Brandenberger and F. Finelli, JHEP 0111, 056 (2001); J. Hwang, astro-ph/0109045 D. H. Lyth, Phys. Lett. B 526, 173 (2002); J. Martin, P. Peter, N. Pinto-Neto, and D. J. Schwarz, Phys. Rev. D 65, 123513 (2002); ibid 67, 028301 (2003), and references therein.

[5] J. Martin and P. Peter, Phys. Rev. D 68, 103517, (2003).

[6] J. Martin and P. Peter, Phys. Rev. Lett. 92, 061301 (2004).

[7] L. D. Landau and E. M. Lifshitz, Quantum Mechanics (Non-relativistic Theory), Butterworth-Heinemann (Oxford, UK, 2000). 